\title{
Parameter Extraction for Electrolyte-Gated Organic Field Effect Transistor Modeling
}

\author{
Deyu Tu, Robert Forchheimer \\ Information Coding, ISY \\ Linköping University \\ SE-581 83, Linköping, Sweden \\ robert@isy.liu.se
}

\author{
Lars Herlogsson, Xavier Crispin, Magnus Berggren \\ Organic Electronics, ITN \\ Linköping University \\ SE-581 83, Linköping, Sweden
}

\begin{abstract}
We present a methodology to extract parameters for an electrolyte-gated organic field effect transistor DC model. The model is based on charge drift/diffusion transport under electric field and covers all regimes. Voltage dependent capacitance, mobility, contact resistance and threshold voltage shift are taken into account in this model. The feature parameters in the model are simply extracted from the transfer or output characteristics of electrolyte-gated organic field effect transistors. The extracted parameters are verified by good agreements between experimental and simulated results.
\end{abstract}

Keywords-electric double layer capacitance; field effect transistors; modeling; parameter extraction

\section{INTRODUCTION}

In electronic device modeling, a simple, straightforward, and efficient method to extract parameters from characteristics is always important for integrated circuit simulation at higher level. In particular, the compact modeling of organic field effect transistors requires the extraction to be as simple as possible, associated with the physically reasonable parameters. Since last two decades, several publications have been devoted to the parameter extraction methodology in organic field effect transistor modeling [1 4]. However, most of them are only focused on organic field effect transistors with insulating dielectric.

Recent progress in organic field effect transistors enables polymer electrolyte as gate dielectric, achieving an operating voltage less than $1 \mathrm{~V}$ [5]. With the capability to form electric double layer capacitors (EDLCs), polymer electrolyte indicates a new generation of organic field effect transistors (OFETs), named electrolyte-gated organic field effect transistors (EGOFETs) or EDLC-OFETs. The formation of EDLCs is a complex electrochemical process, which results nonlinear and voltage-dependent capacitance, and quite crucial to device's performance. We have developed a static model to describe the static electrical behaviors of EGOFETs [6]. The model is based on charge drift in presence of accumulated charges in the channel, covering subthreshold, linear and saturation regimes of the EGOFETs. The charges accumulated are contributed from the field effect caused by electric double layer capacitance dependent voltage biased. Contact barrier dependent on gate voltage and short channel effect are taken

This work was supported by "OPEN" project at the Center of Organic Electronics (COE) at Linköping University, Sweden, funded by the Strategic Research Foundation SSF. into account in this model. In this paper, a simple and efficient approach to extract parameters from DC characteristics of EGOFETs is presented. Good agreements between experimental data and simulation curves are found with the extracted parameters.

\section{EGOFET MODELING}

The static model of EGOFETs is split into three equation to cover subthreshold, linear and saturation regimes, respectively. Based on these base equations, the modifications with contact resistance and threshold voltage shift are given subsequently.

\section{A. Linear Regime}

In this regime, the channel current $I_{D}$ model is express as

$$
\begin{aligned}
I_{D, \text { lin }} & =\frac{W}{L} \mu_{0}\left[C_{0} \frac{V_{G T}^{\gamma+2}-\left(V_{G T}-V_{D}\right)^{\gamma+2}}{\gamma+2}\right. \\
& \left.+C_{i} \frac{V_{G T}^{\gamma+\chi+2}-\left(V_{G T}-V_{D}\right)^{\gamma+\chi+2}}{\gamma+\chi+2}\right]
\end{aligned}
$$

where $W$ is the channel width, $L$ is the channel length, $\mu_{0}$ is the low-field mobility, $C_{0}$ is the voltage-independent of capacitance, $C_{V}$ is the voltage-dependent capacitance, $V_{G T}$ is the gate voltage $V_{G}$ minus the threshold voltage $V_{T}, V_{D}$ is the drain voltage, $\gamma$ is the mobility enhancement factor, and $\chi$ is the voltage-dependent factor of EDLC. Here, the voltage dependent capacitance and mobility are both taken into account in this equation.

\section{B. Saturation Regime}

For saturation regime, a channel length modulation factor $1+\lambda\left(V_{D^{-}} V_{G T}\right)$ is introduced to address a short channel effect in the saturation current equation, where $\lambda$ is the channel length modulation coefficient [7]. Hence, drain current for the saturation regime can be written in this form

$$
\begin{aligned}
I_{D, s a t} & =\frac{W}{L} \mu_{0}\left(C_{0} \frac{V_{G T}^{\gamma+2}}{\gamma+2}+C_{i} \frac{V_{G T}^{\gamma+\chi+2}}{\gamma+\chi+2}\right) \\
& \times\left[1+\lambda\left(V_{D}-V_{G T}\right)\right]
\end{aligned}
$$




\section{Subthreshold Regime}

In the subthreshold regime, the charge transport in the channel is not dominated by drift but by diffusion, since most of the induced charges are trapped there. The drain current can be presented as in [8], with a similar expression to conventional transistors

$$
I_{D, \text { sub }}=\frac{W}{L} \mu_{0} C_{0} V_{S S}^{2} \exp \left(\frac{V_{G T}}{V_{S S}}\right)\left[1-\exp \left(\frac{-V_{D}}{V_{S S}}\right)\right]
$$

where $V_{s s}$ is a voltage parameter, reflecting the steepness of the subthreshold characteristics, which can be approximately estimated with the subthreshold slope [9].

\section{Contact Effect}

Contact resistances for organic field effect transistors are often quite substantial and play an important role in the charge transport. The effective drain voltage $V_{D}$ ', excluded the voltage drop on contact resistance, will be

$$
V_{D}{ }^{\prime}=V_{D}-2 I_{D} R_{C} / L \text {. }
$$

The voltage-dependent contact resistance in organic field effect transistors can be expressed as[10]

$$
R_{C}(V)=R_{C 0} V_{G T}^{-(\gamma+1)},
$$

where $R_{C O}$ is the contact resistance at $V_{G T}=1 \mathrm{~V}$.

\section{E. Threshold Voltage Shift}

The threshold voltage can be modified to

$$
V_{T}(L, V)=V_{T, L}\left(1-\frac{\varsigma}{L}\right)+V_{G} \delta_{V T}
$$

where $\zeta$ is a coefficient, $V_{T, L}$ is the threshold voltage of the long channel transistors without short channel effect, and $\delta V_{T}=\partial V_{T} / \partial V_{G}$. is the sensitivity of voltage bias. In this modification, we have considered the influence of both channel length $[4,11]$ and voltage bias [12] on threshold voltage.

\section{PARAMETER EXTRACTION}

EGOFETs with polymer electrolyte P(VPA-AA) and polymer semiconductor PTTTT were fabricated and characterized as reported[5] to extract model parameters. The devices had $\mathrm{Au}$ source/drain bottom electrodes with channel length $L=2$ to $50.5 \mu \mathrm{m}$ and width $W=1000 \mu \mathrm{m}$. The thickness of spin-coated PTTTT layer was around $~ 30 \mathrm{~nm}$ and P(VPAAA) gate dielectric layer was $100 \mathrm{~nm}$, capped by a Ti gate electrode. The measurements were carried out with a Keithley 4200-SCS semiconductor characterization system in ambient air at room temperature. Here, PTTTT transistors with $L=50.5 / 20.5 / 10.5 / 5.5 / 3.5 / 2 \mu \mathrm{m}$ are presented as example to extract parameters in this model.

\section{A. Extraction of Mobility Enhance Factor $\gamma$}

From the power law dependence of contact resistance on voltage, (5) can be rewritten as

$$
\log R_{C}=\log R_{C 0}-(\gamma+1) \log V_{G T} .
$$

Unlike the method in [4], this equation provides an alternative way to extract the mobility enhance factor $\gamma$. Here, we extract contact resistance at $V_{G}=0.6 / 0.7 / 0.8 / 0.9 / 1 \mathrm{~V}$ with the method discussed below, respectively. Then the $\gamma$ can be calculated from the relationship between $R_{C}$ and $V_{G}$.

The $\log R_{C}$ versus $\log V_{G T}$ is plotted in Fig. 1 and a linear fitting gives a slope of -2.33 . Hence, we can obtain the mobility enhance factor $\gamma$, which is around 1.33, comparable with those conventional organic field effect transistors [9].

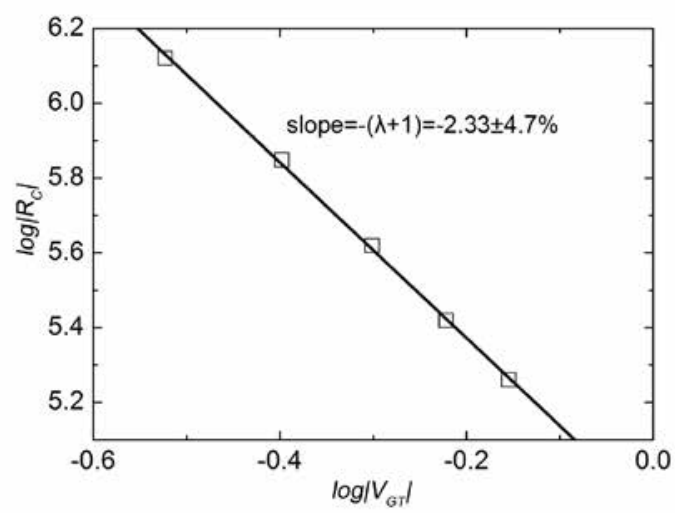

Figure 1. The contact resistance dependence on voltage is graphed with $\log R_{C}$ versus $\log V_{G T}$

\section{B. Extraction of EDLC Factor $\chi$}

To simplify the extraction, we use the saturation equation to obtain the voltage-dependence coefficient $\chi$. In addition, the data are from the transistor with $50.5-\mu m$ channel length, so the short channel effect is negligible. A function $F\left(V_{G}, \chi\right)$, consisting of $V_{G}$ and $\chi$, is written as

$$
F\left(V_{G}, \chi\right)=\frac{\int_{V_{T}}^{V_{G}}\left(\frac{1}{\gamma+2} \frac{\partial I_{D}}{\partial V_{G}} V_{G T}-I_{D}\right) d V_{G}}{\frac{1}{\gamma+2} \frac{\partial I_{D}}{\partial V_{G}} V_{G T}-I_{D}}=\frac{V_{G T}}{\gamma+\chi+3}
$$

In this equation, $F\left(V_{G}, \chi\right)$ becomes a linear function of gate voltage with only another two coefficients $\gamma$ and $\chi$, and the $\gamma$ is 1.33, as extracted above. From the slope of linear fitting in Fig. 2 , presenting the plot of the function $F\left(V_{G}, \chi\right)$, the electric double layer capacitance coefficient $\chi$ is calculated to be 0.67 . 


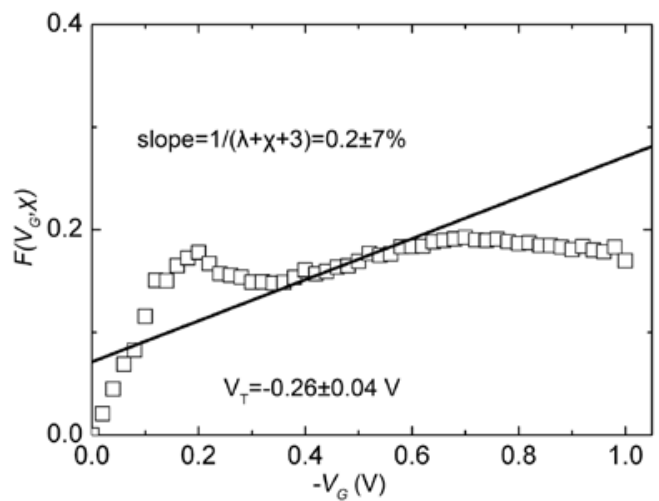

Figure 2. $F\left(V_{G}, \chi\right)$ plot of EGOFET with 50.5- $\mu$ m channel length. The slope of linear fitting gives the coefficient $\chi$.

\section{Extraction of Channel Length Modulation Coefficient $\lambda$}
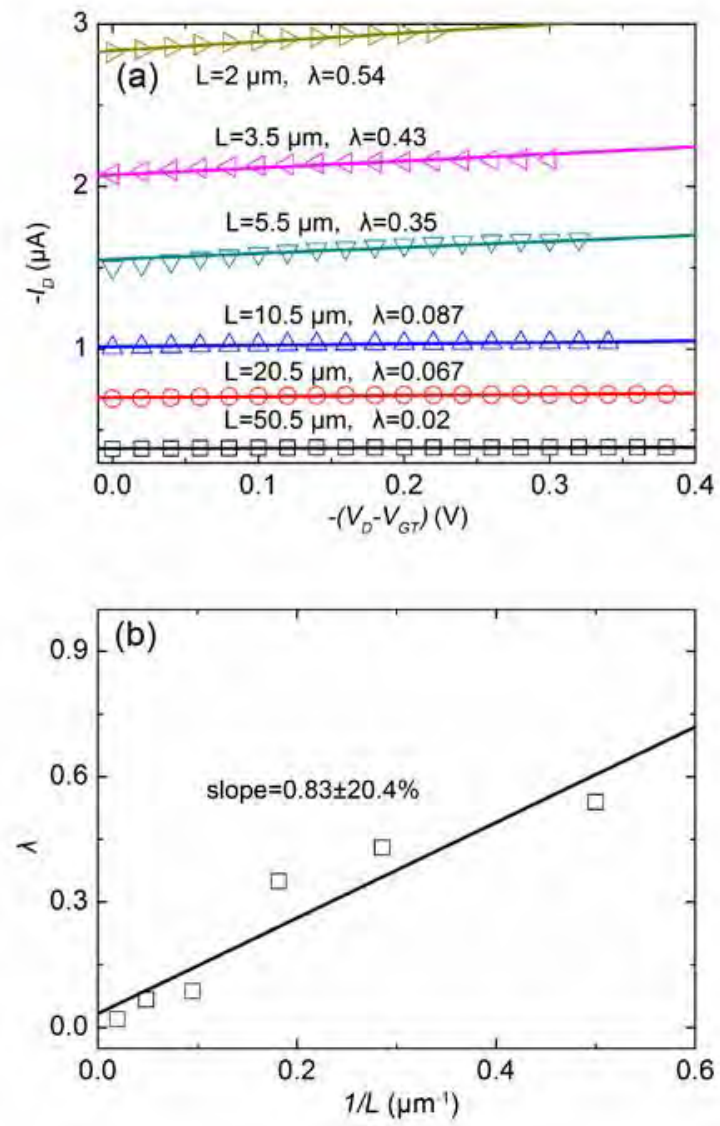

Figure 3. (a) The output curves of EGOFETs with various channel length in saturation regime at $V_{G}=-1 \mathrm{~V}$. (b) The dependence of $\lambda$ on $L$.

From (3), we can see that the increasing current is proportional to the difference of $V_{D}$ and $V_{G T}$ in saturation regime and the slope is $\lambda$. Fig. 3(a) presents the current curves in saturation regime versus $V_{D}-V_{G T}$ and their linear fittings at $V_{G}=-1 \mathrm{~V}$, obtained from transistors with channel length from 2 to $50.5 \mu \mathrm{m}$. The dependence of $\lambda$ on channel length is plotted in Fig. 3(b). Look into Fig. 3(b), we can see the $\lambda$ is dramatically increasing when the channel length is below $5 \mu \mathrm{m}$, indicating a strong short channel effect. From this figure, the coefficient $\lambda$ is denoted as $0.83 / L$, where $L$ is in $\mu \mathrm{m}$.

\section{Extraction of Subthreshold Slope SS}

In subthreshold regime, the subthreshold slope SS is defined as

$$
S S=\frac{V_{G}}{\log I_{D}} .
$$

The SS is extracted from the transfer curves of the $50.5-\mu \mathrm{m}$ channel length transistor, shown in Fig. 4. For this device, the $S S$ is as low as $0.17 \mathrm{~V} / \mathrm{dec}$, implying a fast transition between off state and on state in EGOFETs. In this model, the $S S$ is used to estimate the parameter $V_{S S}=S S / 2$ [5].

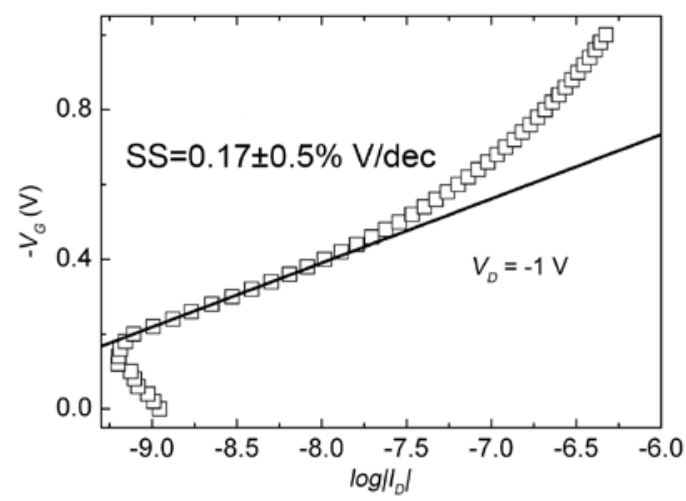

Figure 4. The subthreshold slope is extracted to estimate the parameter $V_{\text {SS. }}$

\section{E. Extraction of Contact Resistance}

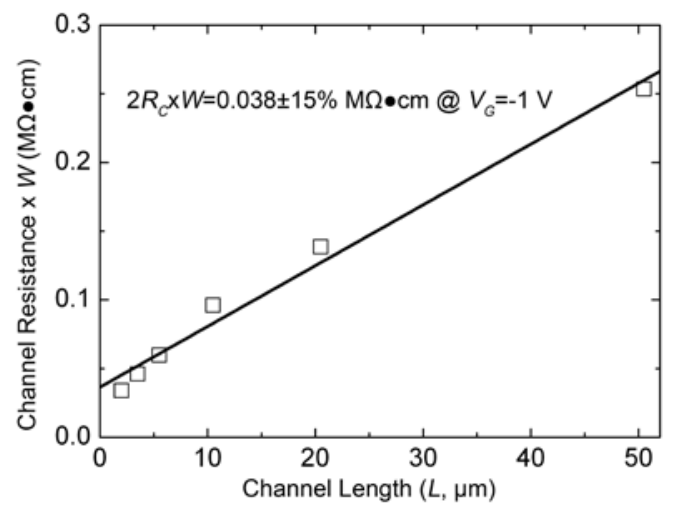

Figure 5. The channel resistance of EGOFETs at $V_{D}=V_{G}=-1 \mathrm{~V}$ with various channel lengths. 
To give the contact resistance referred above, the PTTTT transistors at six channel lengths from 2 to $50.5 \mu \mathrm{m}$ were presented here. The channel resistance of each device at $V_{G}=V_{D}=-1 \mathrm{~V}$ is graphed in Fig. 5 as the hollow squares. A linear fitting of these resistances indicates a contact resistance of $0.19 \mathrm{M} \Omega$ at $V_{G}=V_{D}=-1 \mathrm{~V}$, corresponding $R_{C 0}=0.12 \mathrm{M} \Omega$.

\section{F. Extraction of $V_{T}$ Shift Coefficient $\zeta$ and $\delta_{V T}$}

Here, the threshold voltage of the 50.5- $\mu \mathrm{m}$ channel length transistor is considered to the long channel threshold voltage $V_{T, L}$. The threshold voltage shift $V_{T, L}-V_{T}$ versus $1 / L$ is shown in Fig. 6. From (6), the slope of the linear fitting in Fig. 7 is $V_{T, L} \zeta$, so the threshold voltage shift coefficient $\zeta$ is obtained to be 0.77 . The magnitude of is $\delta_{V T}$ usually very small [5], here we have $\delta_{V T}=0.1$, by linear fitting the threshold voltage extracted at different bias.

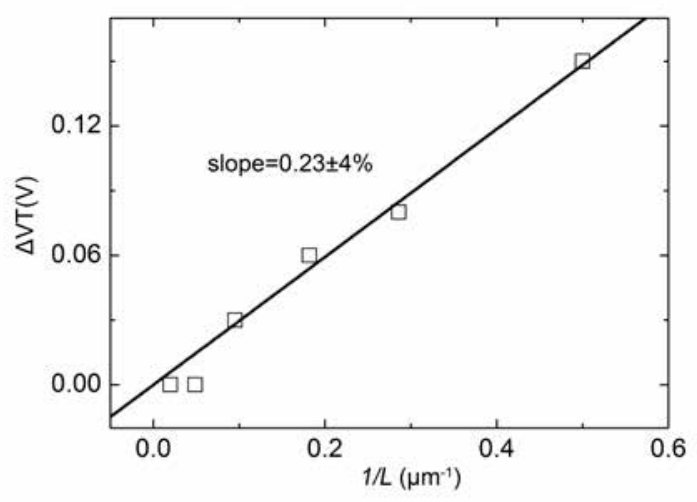

Figure 6. The threshold voltage $V_{T}$ shifts with the channel length $L$, caused by short channel effect.

\section{RESULTS AND DISCUSSION}

An EGOFET with $L=50.5 \mu \mathrm{m}$ is used as example to verify the model parameters extracted as above. The transistor parameters $W=1000 \mu \mathrm{m}$ and $L=50.5 \mu \mathrm{m}$ are given by the experimental conditions, while the parameters $V_{T}=-0.27 \mathrm{~V}, V_{s s}$ $=0.085 \mathrm{~V}, R_{C 0}=0.12 \mathrm{M} \Omega, \gamma=1.33, \chi=0.67, \lambda=0.02, \zeta=0.77$, and $\delta_{\mathrm{VT}}=0.1$ are extracted from the experimental data. Only three parameters are obtained from fitting and they are $C_{0}=5 \mu \mathrm{F} / \mathrm{cm}^{2}$, $C_{V}=1.5 \mu \mathrm{F} / \mathrm{cm}^{2}$ at $\left|V_{G T}\right|=1 \mathrm{~V}$, and $\mu_{0}=0.028 \mathrm{~cm}^{2} / \mathrm{Vs}$. As presented in Fig. 7, the simulated curves given by our model match well with the symbolic circles from experimental data.

\section{CONCLUSION}

In summary, a simple and efficient methodology is presented to extract model parameters for EGOFETs. The static model of EGOFETs is based on charge drift/diffusion in presence of electric double layer capacitor. Taking P(VPAAA)/PTTT EGOFETs as examples, most of important parameters are extracted only from the transfer and output characteristics. With those model parameters extracted, comparisons between experimental data and model theoretical simulations exhibit good agreements.

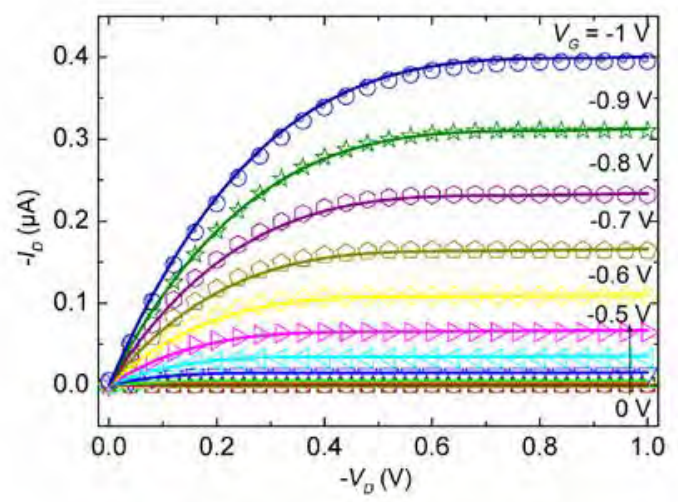

Figure 7. The comparison between calculation and experimental data for output characteristics of EGOFETs.

\section{REFERENCES}

[1] M. Estrada, A. Cerdeira, J. Puigdollers, L. Resendiz, J. Pallares, L. F. Marsal, C. Voz, and B. Iniguez, "Accurate modelling and parameter extraction method for organic TFTs,” Solid State Electron., vol. 49, no. 6, pp. 1009-1016, Jun. 2005.

[2] M. J. Deen, M. H. Kazemeini, and S. Holdcroft, "Contact effects and extraction of intrinsic parameters in poly(3-alkylthiophene) thin-film field-effect transistors,” J. Appl. Phys., vol. 103, no. 12, p. 124 509, Jun. 2008.

[3] P. Lara Bullejos, J. A. Jiminez Tejada, S. Rodriguez-Bolivar, M. J. Deen, and O. Marinov, "Model for the injection of charge through the contacts of organic transistors,” J. Appl. Phys., vol. 105, no. 8, pp. 084516-1084516-8, Apr. 2009.

[4] M. J. Deen, O. Marinov, U. Zschieschang, and H. Klauk, “Organic ThinFilm Transistors: Part II-Parameter Extraction,” IEEE Trans. Electron Devices, vol. 56, no. 12, pp. 2962-2968, Dec. 2009.

[5] L. Herlogsson, X. Crispin, N. D. Robinson, M. Sandberg, O. -J. Hagel, G. Gustafsson, and M. Berggren, "Low-voltage polymer field-effect transistors gated via a proton conductor,” Adv. Mater., vol. 19, no. 1, pp. 97-01, Jan. 2007.

[6] D. Tu, L. Herlogsson, L. Kergoat, X. Crispin, M. Berggren, and R. Forchheimer, "A static model for electrolyte-gated organic field effect transistors,” IEEE Trans. Electron Devices, in press.

[7] L. Li, H. Marien, J. Genoe, M. Steyaert, and P. Heremans, "Compact model for organic thin-film transistor," IEEE Electron Device Lett., vol. 31, no. 3, pp. 210-212, Mar. 2010.

[8] M. Jacunski, M. Shur, A. Owsu, T. Ytterdal, M. Hack, and B. Iniguez, "A short-channel DC SPICE model for polysilicon thin-film transistors including temperature effects,” IEEE Trans. Electron Devices, vol. 46, no. 6, pp. 1146-1158, Jun. 1999.

[9] O. Marinov, M. J. Deen, U. Zschieschang, and H. Klauk, “Organic thinfilm transistors: part I-compact DC modeling,” IEEE Trans. Electron Devices, vol. 56, no. 12, pp. 2952-2961, Dec. 2009.

[10] D. Natali, L. Fumagalli and M. Sampietro, "Modeling of organic thin film transistors: Effect of contact resistances,” J. Appl. Phys., vol. 101, no. 1, pp. 014501, Feb. 2007.

[11] J. N. Haddock, X. Zhang, S. Zheng, Q. Zhang, S. R. Marder, and B. Kippelen, "A comprehensive study of short channel effectsin organic field-effect transistors,” Org. ELectron.,vol. 7, no. 1, pp. 45-54, Jan. 2006.

[12] W. Fichtner and H. W. Pötzl, "MOS Modelling by Analytical Approximations,” Int. J. Electron., vol. 46, no. 1, pp. 33-55, Jan. 1979. 